\title{
Racism, equity and inclusion in research funding
}

\author{
Ying Lia Lia, Hope Bretscher ${ }^{b}$, Rachel A Oliver ${ }^{b}$, Erinma E Ochu ${ }^{c^{*}}$
}

a University College London

${ }^{b}$ University of Cambridge

c Manchester Metropolitan University

\section{Science depends on research funding}

Government funded research grants from United Kingdom Research and Innovation (UKRI) are the lifeblood of our research ecosystem in science, engineering, technology, mathematics and medicine (STEMM). These grants pay the salaries of researchers, support staff and technicians, allow academics to buy consumables and equipment, and cement partnerships, including access to world class facilities. This pre-determines what knowledge is produced. Winning grants is vital to career progression from being a PhD student, to developing independence as an early career researcher, to running your own lab and hiring a research team. Whilst this article recognises the systemic barriers in progression in higher education and STEMM careers ${ }^{1}$ that privilege ${ }^{2}$ 'white' people, we focus on evidence within the grant funding system to consider discrepancies in who is given the opportunity to do research and why this matters.

Funding data recently released by UKRI ${ }^{3}$ highlight the different success rates, grant amounts and experiences of 'Black' and 'ethnic' minority applicants and awardees over the past 5 years, compared to 'white' researchers. When discussing these data, we are mindful that race and ethnicity are long proven to be social constructs ${ }^{4}$ for maintaining power and privilege. We acknowledge that imperial science has played a role in racialised constructions of power ${ }^{5}$, and that data collected using categories such as 'Black, Asian and Minority Ethnic (BAME)' homogenise across different cultural backgrounds ${ }^{6}$. Since, data collection by funders adopts these terms, we employ them to highlight racialised inequity in funding allocation, which damages the economy and society. A weakness of this dataset is that focusing on BAME versus white categories alone, hides anti-Black racism and ignores the experience of those with intersectional identities, across race, gender ${ }^{1,7}$, class, disability ${ }^{8}$ and/ or LGBTQI+ ${ }^{9}$.

Specifically, we focus here on data released by the Engineering and Physical Sciences Research Council ${ }^{10}$ (EPSRC), one of the UKRl's 11 constituent research councils. Many of the trends we present are seen more broadly across UKRl's STEMM-oriented councils. One key observation from the EPSRC data is that for every year of the last five years, lead applicants who identify in funding applications as an 'ethnic minority' have been less successful in their grant applications than those who identify as 'white'. The average success rate is $25 \pm 1 \%$, for 'ethnic minority' researchers as compared to $33 \pm 2 \%$ for 'white' researchers. But what difference do these numbers imply for researchers, in everyday terms?

\footnotetext{
*e.ochu@mmu.ac.uk
} 


\section{FIGURE 1}

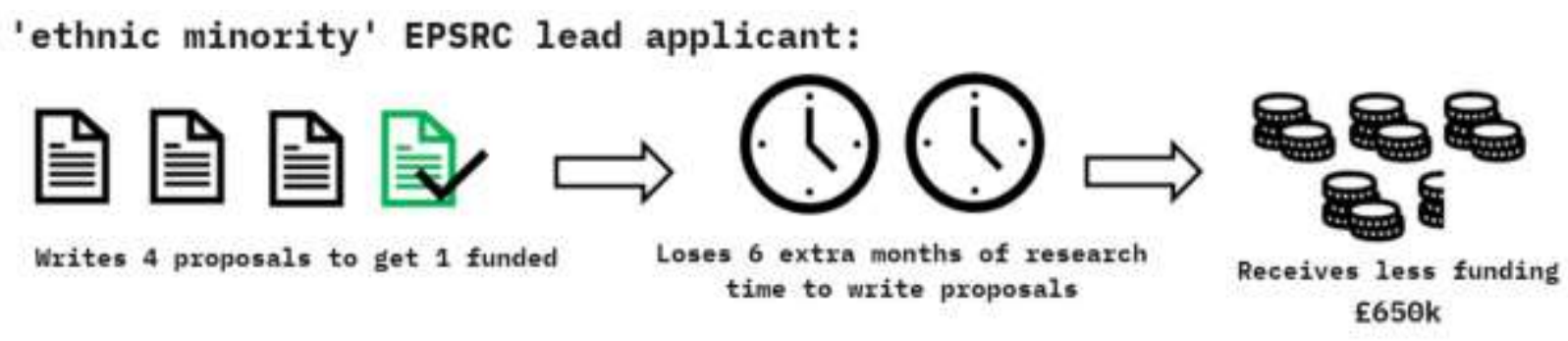

'white' EPSRC lead applicant:

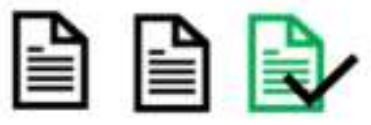

Writes 3 proposals to get 1 funded
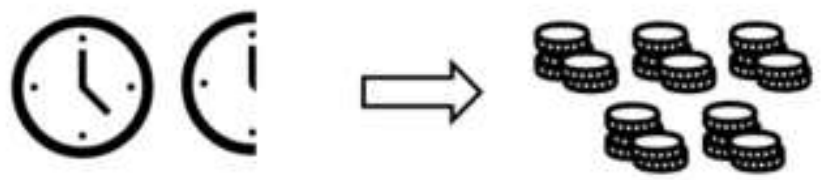

€715k

These data imply (Figure 1) that an 'ethnic minority' researcher needs to write four proposals on average in order to win one grant, compared to a 'white' researcher who writes three. Proposal writing generally takes six months, and equates to lost research time. When 'ethnic minority' researchers do win grants, the average grant award over the past 5 years is approximately $£ 65,000$ lower than for 'white' awardees. This is the equivalent cost of an experienced researcher working in your lab for one year. The resulting underfunding may mean that the 'ethnic minority' researcher achieves fewer published papers, and less impact for their labour. It should be noted that these data on grant value probably underestimate the true scale of the problem since the EPSRC data omit some very large awards, for example for the founding or continuation of research institutes, and we observe that these are won overwhelmingly by older, white men.

The language of 'winning' and 'losing' funding assumes there is a level playing field in the STEMM funding 'game'. This assumption ignores the historical impacts of racism in academia, and in broader society. This is also evidenced by funding data, which indicates that 'ethnic minority' students are less likely to be UKRI-funded than 'white' students ${ }^{11}{ }^{12}$. Likewise at the most senior decision-making levels, 'ethnic minority' scientists are severely underrepresented ${ }^{13}$. This means that senior researchers devising calls for research proposals and judging the resulting applications are not representative of the UK tax paying population, who fund research.

This under-representation creates additional barriers to the success of 'ethnic minority' researchers within their own institutions: racial microaggressions; lack of support for proposal development; and the privileging of 'white' researchers in both job promotions and the institutional sifting processes that determine who is allowed to apply for grants. Together, this can lead to many minoritised researchers leaving academia ${ }^{14}$ or remaining precariously employed on short term contracts ${ }^{15}$. For those who stay, failure to 'win' on the skewed playing field of the funding game, leads to a cycle of reduced opportunities for research career progression, as shown in Figure 2. While some scientists have found ways of circumnavigating 
or flipping these barriers ${ }^{16}$, to drive innovation alongside community or industrial partners, the 'make it or break it' role of funders and their funding cycles remains a recurring theme. There is a lack of recognition of the ways in which minoritised researchers, carve out alternative career pathways, take on unacknowledged Equality, Diversity and Inclusion work to reduce institutional barriers ${ }^{11}$ and carry out more equitable and inclusive research that benefits society ${ }^{17}$.

\section{FIGURE 2}

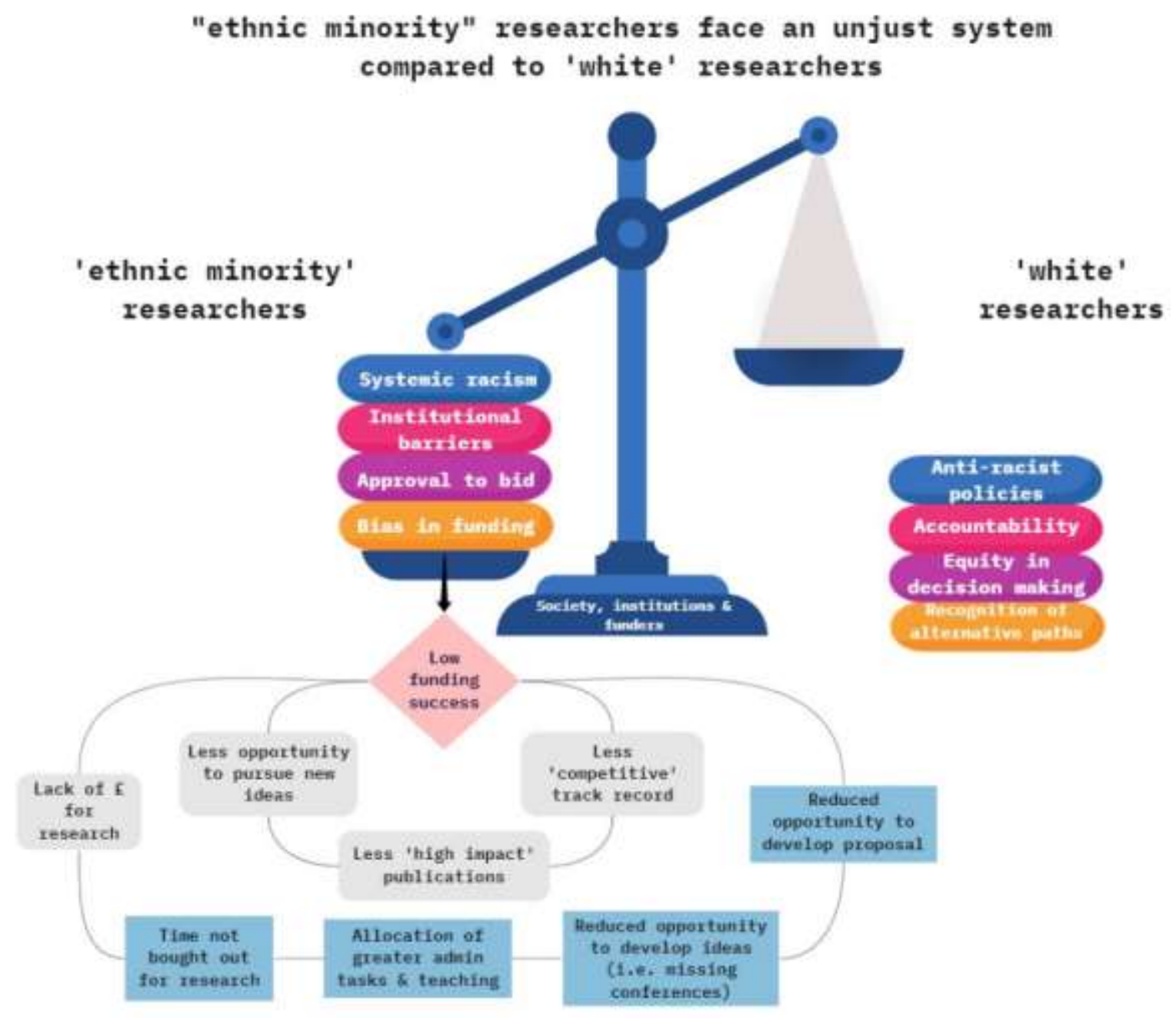

\section{Funding equity benefits science \& innovation}

Promoting equity via institutional and funder policy leads to better outcomes. How research is funded and who gets funded to carry out research has drastic impacts on society. The voices and ideas that are excluded and the science that is underfunded ${ }^{17,18}$ cause harm to minoritised communities. In the COVID-19 pandemic, minoritised communities, specifically, African, Carribbean, Bangladeshi and Pakistani people have died at a much higher rate than 'white' people $^{19}$, an issue which was addressed by UKRI in a specific call for research proposals. A Black women's collective, orchestrated by Dr Addy Adelaine, who specialises in 'inclusive accountability', investigated who was funded as an outcome of this call, which failed to award 
funding to Black researchers, in spite of many applying ${ }^{20}$. As a result, the funded projects were highly slanted towards genetic and biological factors, an approach which is scientifically contested and fails to account for the systemic and social factors which Black researchers had proposed to investigate. These abuses of power and privilege not only prevent Black communities from generating effective solutions but also risk further reducing trust in science. Similar issues are prevalent within environmental science and climate change research ${ }^{21,22}$. Crucially, whilst some collaborative initiatives seek to overturn this trend, existing inequities often place the burden on resource-stretched community practitioners ${ }^{23}$. An important step is acknowledging that systemic inequity and racism exist, to begin to redress the balance and reap societal benefits.

Increasing the diversity of the workforce is known to improve outcomes in many sectors ${ }^{24,25,26,27}$. One recent study used automated text-based analysis to look for markers of innovation across 1.2 million PhD theses published in the USA between 1977 and $2015^{28}$. The study found that minority scientists are more innovative than their majority counterparts, but that they receive less reward for their new ideas and inventions. This suggests that ethnic minority researchers may well be more innovative than their white peers - but they are being held back from success by the funding system. This suppresses innovations which could create a stronger and more inclusive economy.

\section{What can we do?}

It is vital to consider and report on the diversity of those framing and judging research proposals. The Haldane principle states that decisions about what to spend research funds on should be made by researchers rather than politicians. This principle, coupled with the concept of peer review (where researchers' proposals and outputs are judged by their peers) notionally underpin our entire research funding system. However, Black and minority ethnic researchers are largely not judged by their Black and minority ethnic peers. EPSRC, for example, convenes expert panels to make decisions on which proposals should be funded, but only $8 \%$ of EPSRC panel members and 5\% of EPSRC panel chairs identify as an ethnic minority, whilst ethnic minority researchers make up $20 \%$ of the EPSRC researcher cohort ${ }^{29}$. Funders have a responsibility to ensure panels are culturally diverse, that panel members are adequately trained, and funding decisions or feedback are not racially prejudiced.

In order to ensure that research proposals from Black and minority ethnic scientists actually reach this vital peer review stage, funders could mandate that institutions meet minimum requirements for removing the barriers experienced by Black and ethnic minority researchers, which could be evidenced by Race Equality Charter accreditation. Accredited institutions should demonstrably monitor and boost the number of minoritised researchers applying for funding and improve the support they are offered.

When the National Institute for Health Research (NIHR) introduced incentives to encourage institutions to achieve Athena Swan accreditation for dismantling barriers to women's progress, the number of women in mid-level leadership positions and the proportion of funding going to women increased ${ }^{30}$. This demonstrates the effectiveness of this type of approach, which 
unfortunately the government has recently banned research funders from following ${ }^{31}$. Nonetheless, it is vital that meaningful incentives are established by UKRI and other research funders to increase the diversity of both those who receive funding and those who make funding decisions. This must be achieved within a culture of increased transparency and accountability. Some funders, such as Wellcome, have appointed an anti-racism expert group.

More radically, since the Haldane principle nominally encourages researchers to decide on the direction of scientific research and training, we can consider measures which empower every researcher to influence funding decisions, rather than just a privileged few. This would require a substantial overhaul of our funding processes. Novel approaches such as the "Universal Basic Research Grant"32 (in which all researchers receive at least some minimum financial support to explore their ideas) or full ${ }^{33}$ or hybrid ${ }^{34}$ lottery systems, could potentially reduce the impact of racism on our scientific systems, as long as pitfalls such as Institutional gatekeeping of access to such schemes are avoided.

Given the growing need for research and innovation to address societies' biggest challenges, from pandemics to the climate crisis to systemic abuses of power, change is urgently needed. Individual researchers, institutions, funders and the government can all play a role by committing to change, addressing inequity and taking action together. 


\section{$\underline{B i o s}$}

Dr Ying Lia Li is a physicist working on quantum sensing at University College London. Between 2017-19 she ran the UCL Women in Physics Group \& is currently a member of the UCL Race Equality Steering Group. She is also the Founder of Zero Point Motion, an early stage start-up developing chipscale optical inertial sensors for navigation.

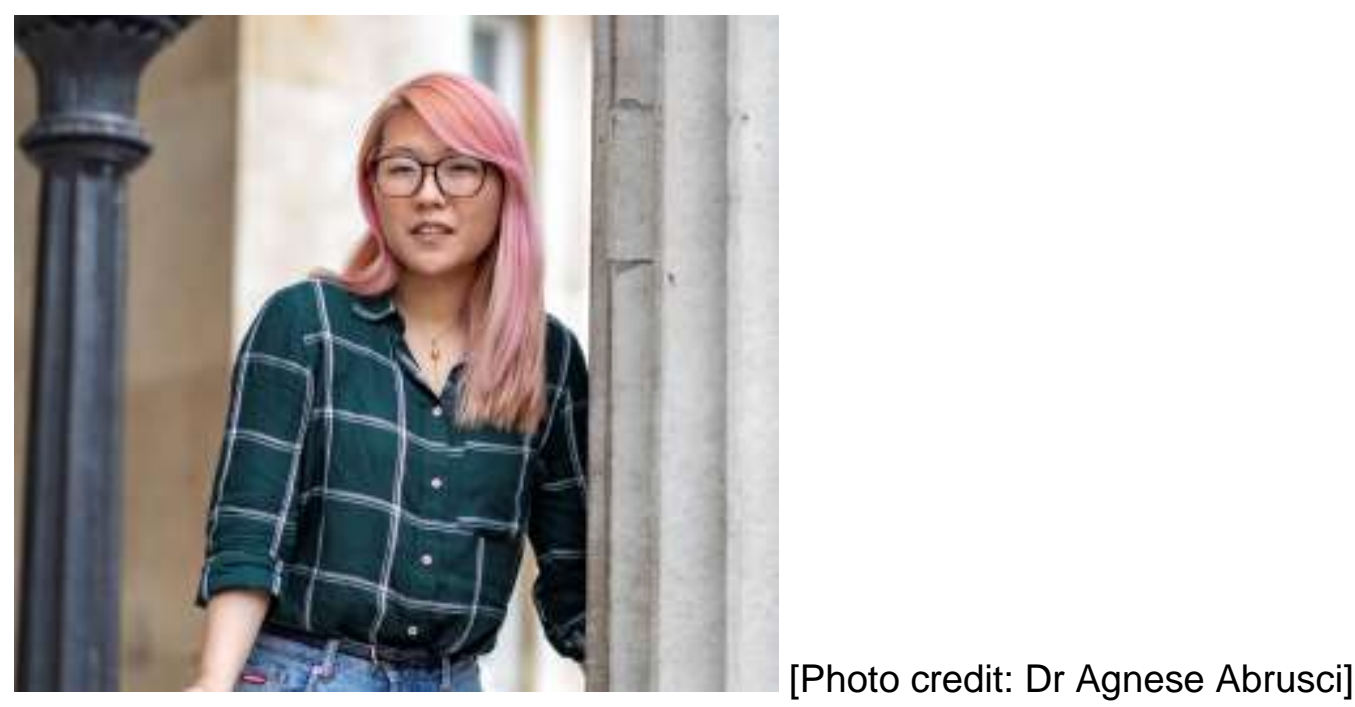

Hope Bretscher is a condensed matter physicist and activist. She is currently finishing her PhD at the University of Cambridge and moving to the Max Planck Institute for the Structure and Dynamics of Matter.

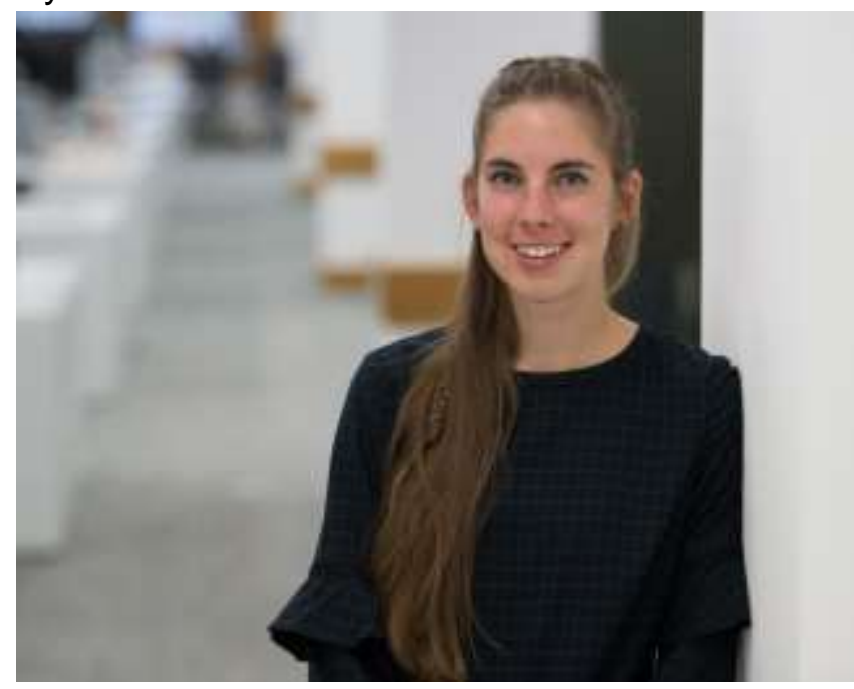


Professor Rachel Oliver is a materials scientist at Cambridge University studying materials for photonic and electronic devices. In 2020, she was named as one of the Top 50 Women in Engineering by the Women in Engineering Society. She is an Equality and Diversity Champion of Cambridge's School of Physical Sciences and a founding member of The Inclusion Group for Equity in Research in STEMM (TIGERS).

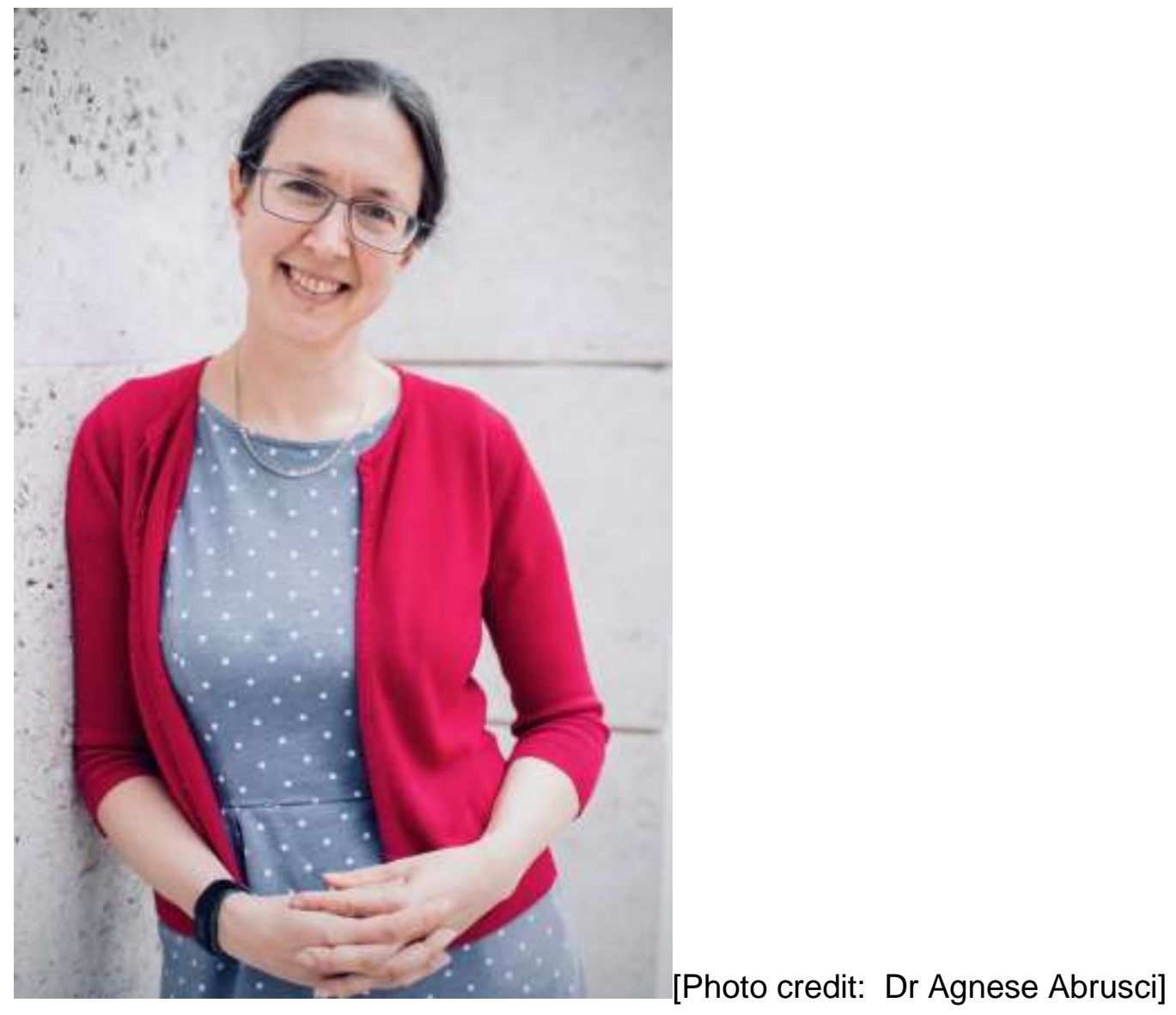


Dr Erinma Ochu, MBE is interim Director of Engaging Environments, a NERC climate solutions initiative to build capacity for inclusive research. They are a JUST AI Visiting Fellow at The Ada Lovelace Institute, decolonising Al in service to racial justice. A neuroscientist and filmmaker, Erinma is Senior Lecturer in Digital Media and Communications in the iSchool at Manchester Metropolitan University.

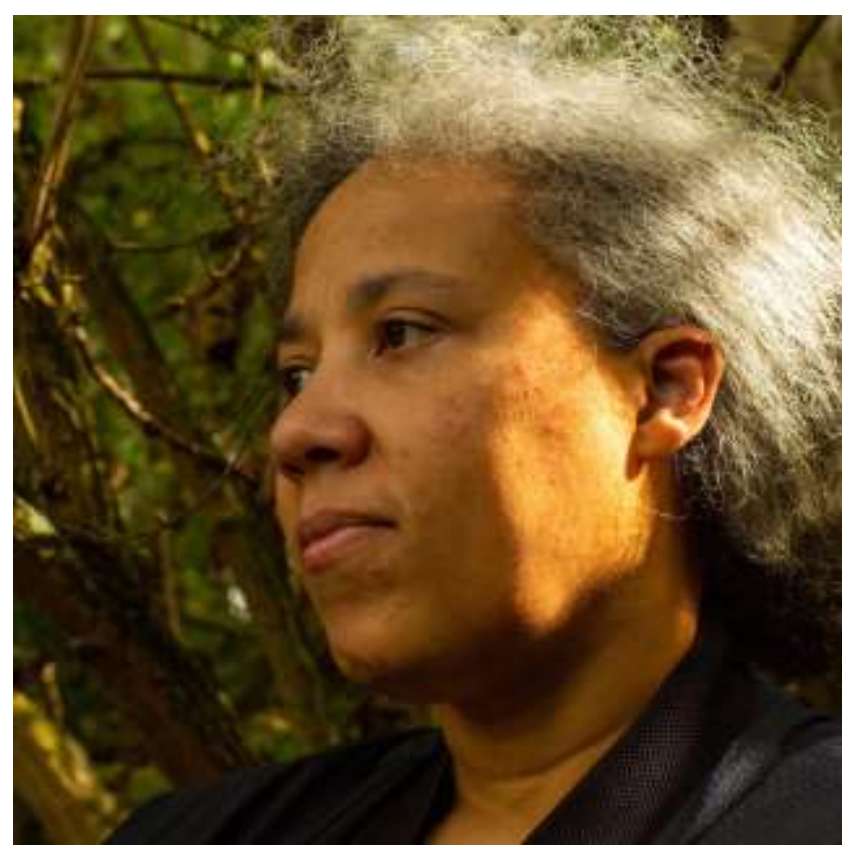


${ }^{1}$ Rollock N 2019 Staying Power https://www.ucu.org.uk/media/10075/StayingPower/pdf/UCU_Rollock_February_2019.pdf

${ }^{2}$ Bhopal B 2018 White Privilege: The Myth of a Post-Racial Society. Polity Press.

3 https://www.ukri.org/our-work/supporting-healthy-research-and-innovation-culture/equalitydiversity-and-inclusion/diversity-data/

4 Du Bois WEB [1940] 1994 Dusk of Dawn: An Autobiography of a Race Concept. New

Brunswick, NJ: Transaction

${ }^{5}$ Saini A 2019 Superior. Fourth Estate

${ }^{6}$ Very shortly before the submission of this article, UKRI released a new data set in which the impact of ethnicity on funding rates is reported with greater granularity than the binary division previously used between 'white' and '"ethnic minority'. However, this dataset does not separate funding for STEMM research from other streams, and has yet to be fully analysed.

7 Jebsen J et al. 2020 A Review of Barriers Women Face in Research Funding Processes in the UK https://psyarxiv.com/27mdz/

8 TigerInSTEMM 2020 Accessibility in STEM: Barriers facing disabled individuals in research funding processes https://osf.io/uzsdk/

9 TigerInSTEMM 2020 Barriers LGBTQI+ People Face in the Research Funding Processes https://osf.io/dnhv8/

${ }_{10}$ https://www.ukri.org/wp-content/uploads/2020/10/UKRI-020920-PIApplicationAmountAndAwardValue.xlsx

${ }^{11}$ Uchegbu I. $2020 \mathrm{https}: / /$ youtu.be/VyC3Qr 5CUY [replace with ljeoma's article if possible]

${ }^{12}$ Williams P. et al. 2019 The Broken Pipeline - Barriers to Black PhD Students Accessing Research Council Funding, https://leadingroutes.org/mdocs-posts/the-broken-pipeline-barriers-to-black-studentsaccessing-research-council-funding

13 University and College Union 2016 The experiences of Black and Minority Ethnic staff in further and higher education https://www.ucu.org.uk/media/7861/The-experiences-of-black-and-minority-ethnic-staffin-further-and-higher-education-Feb-16/pdf/BME_survey_report_Feb161.pdf

${ }^{14}$ Bhopal K, Brown H, Jackson J (for the Equality Challenge Unit) 2015 Academic flight: how to encourage black and minority ethnic academics to stay in UK higher education https://www.ecu.ac.uk/wpcontent/uploads/2015/03/ECU_Academic-flight-from-UK-education_RR.pdf

$15 \mathrm{UCU}, 2019 \mathrm{Job}$ insecurities in universities: the scale of the problem https://www.ucu.org.uk/media/10502/Job-security-in-universities---the-scale-of-theproblem/pdf/ucu_casualisation-in-he_graphic_oct191.pdf

16 "Aiming Higher: Race, Inequality and Diversity in the Academy", Runnymede Perspectives, Edited by Claire Alexander and Jason Arday, 2015

${ }^{17}$ Edge D. 2020 'Scientific Racism' and structural inequalities: Implications for researching Black mental health

${ }^{18}$ Hoppe T.A. et al. 2019 Topic choice contributes to the lower rate of NIH awards to AfricanAmerican/black scientists, Science Advances Volume 5, Article No. eaaw7238.

19 Lawrence D. 2020 An Avoidable Crisis: The disproportionate impact of Covid-19 on Black, Asian and minority ethnic communities, https://www.lawrencereview.co.uk/

${ }^{20}$ Adelaine et al. 2020 Knowledge Is Power-An Open Letter To UKRI, https://www.researchprofessionalnews.com/rr-news-uk-views-of-the-uk-2020-8-knowledge-is-power-anopen-letter-to-ukri/

${ }^{21}$ Pearson AR \& Schuldt JP 2014 Facing the diversity crisis in climate science Nature Climate Change Volume 4, Pages1039-1042

22 Thomas A \& Haynes R 2020 Black Lives Matter: the link between climate change and racial justice https://climateanalytics.org/blog/2020/black-lives-matter-the-link-between-climate-change-and-racialjustice/

${ }^{23}$ Runnymede Trust 2018 Finding Common Cause: Building research collaborations between universities and Black and minority ethnic communities 
https://www.runnymedetrust.org/uploads/publications/Runnymede\%20Common\%20Cause\%20briefing\% 20FINAL.pdf

${ }^{24}$ Levine SS et al. 2014 Ethnic diversity deflates price bubbles" PNAS Volume 111, pages 1852418529.

${ }^{25}$ Hunt $\mathrm{V}$ et al. 2018 Delivering through Diversity, https://www.mckinsey.com/business-

functions/organization/our-insights/delivering-through-

diversity?utm_source=UCL\%20\%28Internal\%20Communications\%29\&utm_medium=email\&utm_campai gn=10333188_Provost\%27s\%20View\%3A\%20Equality\%2C\%20Diversity\%20and\%20Inclusion\%20at\%2 OUCL\%20\%E2\%80\%93\%20challenging\%20unacceptable\%20behaviour\%20and\%20attitudes\&utm_cont ent=McKinsey\&dm_i=UAA,65H50,HP7E2G,O86HP,1\#

${ }^{26}$ AIShebli BK, Rahwan T \& Woon WL 2018 The Preeminence of Ethnic Diversity in Scientific Collaboration Nat Commun Volume 9, page 5163.

${ }^{27} \mathrm{https}$ ://www.raeng.org.uk/diversity-in-engineering/business-benefits-key-facts/the-business-case-fordiversity

${ }^{28}$ Hofstra B et al. 2020 The Diversity-Innovation Paradox in Science PNAS Volume 117, pages 92849291.

29 https://epsrc.ukri.org/files/funding/edi/epsrc-peer-review-diversity-data-narrative-and-tables-april-2019$\mathrm{pdf} /$

${ }^{30}$ Kalpazidou Schmidt E et al. 2020 Understanding the Athena SWAN award scheme for gender equality as a complex social intervention in a complex system: analysis of Silver award action plans in a comparative European perspective. Health Res Policy Sys Volume 18, Article number 19 https://doi.org/10.1186/s12961-020-0527-x.

${ }^{31}$ Reducing bureaucratic burden in research, innovation and higher education https://www.gov.uk/government/publications/reducing-bureaucratic-burdens-higher-education/reducingbureaucratic-burdens-on-research-innovation-and-higher-education

32 Payne D 2019 The universal basic research grant: funding research for the 21st century https://wonkhe.com/blogs/the-universal-basic-research-grant-funding-research-for-the-21st-century/

${ }^{33}$ Adam D 2019 Science funders gamble on grant lotteries Nature Volume 575, Pages 574-575

${ }^{34}$ Schwartz B 2019 Do College Admissions by Lottery https://behavioralscientist.org/do-collegeadmissions-by-lottery/ 